\title{
Rol Modulador de la Oxitocina en la Interacción Social y el Estrés Social ${ }^{*}$
}

\section{Oxytocin's Modulator Role in Social Interaction and Social Stress}

DOI: $10.11144 /$ Javeriana.upsy15-5.rmoi

Recepción: 11 Septiembre 2016 | Aprobación: 16 Noviembre 2016

\author{
Stefani Florez Acevedo ${ }^{\mathrm{a}}$ \\ Universidad de Los Andes, Colombia \\ ORCID: http://orcid.org/0000-0002-8786-0614 \\ Luis Fernando Cardenas Parra \\ Universidad de Los Andes, Colombia
}

aAutor de correspondencia. E-mail:
stefanipfa@gmail.com

Para citar este artículo: Florez-Acevedo, S. \& Cardenas, F. P. (2016). Rol Modulador de la Oxitocina en la Interacción Social y el Estrés Social. Universitas Psychologica, 15(5). http:// dx.doi.org/10.11144/Javeriana.upsy15-5.rmoi

\section{RESUMEN}

La Oxitocina es un neuropéptido conocido por facilitar funciones del sistema nervioso periférico, relacionadas específicamente con el sistema reproductivo. Sin embargo, en las últimas décadas se ha reconocido la función moduladora de la Oxitocina en el comportamiento social, a través de su liberación en el sistema nervioso central. Así mismo, estudios han mencionado que la Oxitocina es un potencial ansiolítico cuando un individuo ha sido sometido a estrés social. Por lo tanto, el objetivo de esta revisión es presentar una caracterización de la Oxitocina y su relación con distintas formas de interacción social y el estrés social; a través de los resultados presentados en distintos estudios, tanto en modelos animales como en humanos. Además, se intenta mostrar la importancia de continuar con el estudio de la Oxitocina, dados los posibles vacíos teóricos y experimentales existentes, teniendo en cuenta las potenciales cualidades ansiolíticas de esta hormona.

Palabras clave

Oxitocina; Interacción social; Estrés social; Ansiedad.

\begin{abstract}
Oxytocin, a neuropeptide, is known to allow peripheral nervous system functions related specifically to the reproductive system. However, the modulatory function of Oxytocin in social behavior has been recognized in the last decades through its release in the central nervous system. Likewise, some studies have mentioned that Oxytocin is a promising anxiolytic when an individual has been exposed to social stress. Therefore, the objective in this review is to show a characterization of Oxytocin and its relationship with both social interactions and social stress, through results of studies in both animals and humans. Also, this review intends to show the importance of furthering the study of Oxytocin due to the theoretical and experimental voids in its current research, knowing the potential anxiolytic qualities of this hormone.
\end{abstract}

Keywords

Oxytocin; Social Interaction; Social Stress; Anxiety. 


\section{Introducción}

La Oxitocina (OT) es una hormona nanopéptida formada por nueve aminoácidos: cisteína-tirosina-isoleucinaglutamina-asparagina-cisteína-prolina-leucinaglicina con un puente de azufre entre las dos cisteínas (Lee, Macbeth, Pagani \& Scott, 2009). Ésta hormona ha sido conocido desde principios del siglo XX por ejerce funciones a nivel periférico: contracciones uterinas y lactancia. Sin embrago, durante las décadas de los 80 y 90 su atractivo investigativo aumentó cuando se evidenció que además de sus efectos periféricos, la OT intervenía a nivel central (Lee et al., 2009) modulando comportamientos como el materno (Pedersen, Caldwell, Peterson, Walker, \& Mason, 1992; Pedersen \& Prange, Jr., 1985), el reproductivo (Caldwell, Walker, Pedersen \& Mason, 1993) y el de vínculos sociales Caldwell et al., 1993; Carter, DeVries, \& Getz, 1995). Éstas áreas de estudio dieron paso a la gran variedad de investigación del siglo XXI que actualmente se lleva a cabo sobre el efecto de la OT en distintos tipos de comportamientos sociales como el agresivo (Neumann, 2007; Bosch, Meddle, Beiderbeck, Douglas, \& Neumann, 2005; Litvin, Murakami \& Pfaff, 2011); el emparejamiento o conformación de pareja (Schneiderman, Zagoory-Sharon, Leckman \& Feldman, 2012; Young, Lim, Gingrich \& Insel, 2001); el parental (Lee et al., 2009 ; Strathearn, Iyengar, Fonagy \& Kim, 2012; Naber et al., 2010) y el reproductivo (Lee et al., 2009).

Teniendo en cuenta el relevante rol de la OT en las diferentes formas de comportamiento social mencionadas, esta revisión tiene por objetivo presentar una caracterización de la OT y su relación con el comportamiento social y el estrés social, a través de los resultados presentados en distintos estudios, tanto en modelos animales como en humanos. Así mismo, se presenta la importancia de continuar con el estudio de la OT dados los posibles vacíos teóricos y experimentales existentes, teniendo en cuenta, que este neuropéptido podría tener propiedades ansiolíticas prometedoras.

\section{Revisión de las Propiedades Fisiológicas de la Oxitocina}

El gen de la OT está localizado en el cromosoma 2 en ratones y en el cromosoma 20 en humanos (Lee et al., 2009). La OT es secretada por la neurohipófisis, la cual es activada por proyecciones desde el hipotálamo (Bethlehem, van Honk, Auyeung \& Baron-Cohen, 2013) para actuar en el sistema nervioso periférico (SNP) y en el sistema nervioso central (SNC). En el SNP la Oxitocina es metabolizada en el útero, la placenta, el amnios, los testículos, el páncreas, los riñones y el corazón (Gimpl \& Fahrenholz, 2001). Debido a que la OT liberada en el sistema periférico no puede cruzar la barrera hemato-encefálica en altas concentraciones, la posibilidad de cambios comportamentales relevantes es baja (Neumann, 2008), por lo tanto, solo la OT liberada en el SNC originará efectos significativos en el comportamiento.

La síntesis de OT en el SNC se produce por la expresión predominante del gen de la OT en neuronas localizadas en los núcleos paraventricular (NPV) y supraóptico (NSO) del hipotálamo (Kiss \& Mikkelsen, 2005). La liberación de la OT se realiza por el NSO a través de neuronas magnocelulares que se proyectan a la neurohipófisis (Bethlehem et al., 2013); mientras que proyecciones axonales de pequeñas neuronas parvocelulares del NPV inervan a la amígdala, el hipocampo, el núcleo accumbens y el septo lateral (Ross \& Young, 2009).

Similar a los neurotransmisores clásicos como la dopamina, serotonina, GABA o acetilcolina, la OT está conservada en vesículas que pueden ser liberadas por estimulación eléctrica (Ludwig \& Leng, 2006) a la hendidura sináptica; pero a diferencia de los neurotransmisores clásicos, la OT se puede difundir a través del espacio extracelular (Ross \& Young, 2009) debido a su vida media (aproximadamente 20 minutos en el cerebro); lo que implica una degradación lenta y no presencia de restricciones sinápticas espaciales (Ludwig \& Leng, 2006). Para evocar la liberación dendrítica de la OT, las neuronas de OT del NPV y del NSO expresan el receptor Melanocortina-4 (MC4) que al unirse con la 
hormona alfa melanocito estimulante (\#MSH) induce la liberación de Oxitocina de las dendritas de las neuronas magnocelulares (Sabatier, 2006). Específicamente, la liberación de OT en el NPV se difunde a otras regiones del cerebro y así tiene efectos sobre el comportamiento (Ludwig \& Leng, 2006).

\section{Receptor de OT y su distribución en el sistema nervioso}

Hasta la fecha sólo un receptor de Oxitocina (OTR) ha sido clonado (Bales \& Perkeybile, 2012). Éste se caracteriza por tener siete dominios transmembrana (Manning et al., 2012) distribuidos en 3 intrones y 4 exones y está localizado en el cromosoma 3 (3p25) (Vrachnis, Malamas, Sifakis, Deligeoroglou, \& Iliodromiti, 2011). La distribución de los OTR se puede dividir en dos grupos: OTR en el sistema nervioso periférico y OTR en el sistema nervioso central. En el sistema nervioso periférico el OTR se encuentra en el útero, la placenta, las glándulas mamarias, el amnios, el corazón, el páncreas y el hígado (Gimpl \& Fahrenholz, 2001; Kiss \& Mikkelsen, 2005). La distribución de los OTR en el sistema nervioso central ha sido clasificada por diferentes autores, dependiendo de la especie y las estructuras cerebrales. En modelos animales, específicamente en ratas Yoshimura, Kimura, Watanabe, \& Kiyama (1996) clasificaron el OTR en dos grupos: receptores que se expresan de forma transitoria y receptores que se expresan de forma constante y abundante. Los OTR del primer grupo se encuentran distribuidos en el caudado-putamen (CP), corteza cingulada (CC), núcleo anterior talámico y el área tegmental ventral (VTA); mientras que los OTR del segundo grupo se encuentran en: el núcleo olfatorio anterior, base del núcleo de la estría terminal (BNST) y el núcleo ventromedial del hipotálamo (Yoshimura et al., 1996); sin embargo, Lee et al. (2009) y Veinante $\&$ Freund-Mercier (1997) reportaron en sus estudios histoautorradiográficos, que los OTR se distribuían de forma predominante en el núcleo de la estría terminal (BST) lateral y supracapsular y la parte medial y central de la amígdala. Por su parte, Ostrowski en 1998 planteó una nueva clasificación de acuerdo a las regiones implicadas en: los comportamientos reproductivos relacionados a los esteroides, los comportamientos maternos, en los procesos de memoria y aprendizaje y en procesos motivacionales o de refuerzo (Viero et al., 2010). Esta clasificación indica cómo los OTR están ampliamente distribuidos en el sistema límbico, el hipotálamo y el tallo cerebral (Ostrowski, 1998).

A diferencia de la identificación de los OTR en modelos animales, Loup, Tribollet, DuboisDauphin, \& Dreifuss (1991) utilizaron técnicas autorradiográficas in vitro con tejidos de la médula espinal y el cerebro de doce humanos para identificar los lugares predominantes de la unión de la Oxitocina, exclusivamente en los humanos. Como resultado encontraron que los núcleos basales de Meynert, el núcleo de la rama vertical de la banda diagonal de Broca, la parte ventral del núcleo septal lateral, el área hipotalámica preóptica anterior, el área hipotalámica posterior, el globo pálido y el pálido ventral, hacen parte de regiones donde la OT cumple su papel de ligando, afirmando que ella podría actuar como neurotransmisor o neuromodulador en el sistema nervioso central, tal como lo habían indagado en su estudio anterior (Loup, Tribollet, DuboisDauphin, Pizzolato, \& Dreifuss, 1989).

\section{Farmacología de la OT: agonistas y antagonistas}

Los agonistas y antagonistas de OT son moléculas que se han desarrollado como herramientas farmacológicas para tratamientos terapéuticos (Vrachnis et al., 2011) tanto en el sistema nervioso periférico como en sistema nervioso central (Manning et al., 2008). Por medio de la técnica de bioensayos se han identificado cuatro péptidos análogos que son más potentes o selectivos que la OT: [Thr4]OT; HO[Thr4]OT; [Thr4, Gly7]OT y HO[Thr4, Gly7]OT (Manning, Lowbridge, Sawyer, \& Haldar, 1976; Lowbridge, Manning, Haldar, 
\& Sawyer, 1977). Dentro de este grupo de agonistas, el [Thr4, Gly7]OT ha sido el agonista selectivo más utilizado (Manning et al., 2012). Como agonistas no peptídicos se encuentran el WAY-267464 desarrollado por Pfizer (Manning et al., 2012), el cual ha demostrado alta afinidad y potencia como agonista selectivo de OTR y ha demostrado efectos ansiolíticos similares a la OT (Ring et al., 2010).

Como función opuesta de los agonistas de OT, los antagonistas han sido sintetizados para retardar el trabajo de parto, también conocidos como agentes tocolíticos (Vrachnis et al., 2011). Dentro del grupo de los antagonistas peptídicos se encuentra el d[DTyr(Et)2,Thr4]OVT, conocido comercialmente como Atosiban (Manning et al., 2012) y caracterizado por tener afinidad también con los receptores de V1A de vasopresina (AVP) y metabolizarse de forma rápida (Viero et al., 2010). Estudios recientes demostraron que este antagonista puede aumentar el éxito de embarazos de mujeres que han fallado de forma recurrente a fecundación in vitro como tratamiento de la infertilidad y así mismo mejorar el tratamiento de transferencia de embriones (Pierzynski, 2011; Pierzynski, Reinheimer, \& Kuczynski, 2007). Al ser utilizado como tocolítico, el Atosiban presenta algunas desventajas como por ejemplo (1) una biodisponibilidad limitada, (2) requiere de administración parenteral (intradémica, subcutánea, Intramuscular o intravenosa); (3) baja afinidad a los OTR y (4) al tener afinidad con los receptores de la Vasopresina (específicamente con el V1A) causa efectos secundarios (Vrachnis et al., 2011).

Teniendo en cuenta la baja afinidad del Atosiban, Manning et al. (2012) compararon varios antagonistas peptídicos que tienen alta afinidad con los receptores humanos como por ejemplo el d(CH2)5[Tyr(Me)2]OVT; desGly-NH2,d(CH2)5[Tyr(Me)2, Thr4]OVT; d(CH2)5[Tyr(Me)2, Thr4,Tyr-NH29 ]OVT y desGly-NH2,d(CH2)5 [D-Tyr2, Thr4]OVT, y encontraron que el desGly-NH2,d(CH2)5 [DTyr2, Thr4]OVT es el antagonista más selectivo. La administración central de este antagonista selectivo se caracteriza por su efecto como bloqueador de los componentes receptivos y proceptivos del comportamiento sexual en hembras (Pedersen \& Boccia, 2002) y por bloquear comportamientos de ansiedad generados por el aumento de la actividad del eje hipotalámico-pituitario-adrenal (HPA) tanto en machos como en hembras (Neumann, Wigger, Torner, Holsboer, \& Landgraf, 2000). Otro antagonista peptídico con alta afinidad en humanos es el Barusiban, el cual se caracteriza por tener mayor afinidad por el receptor OTR que por los receptores para V1A, a diferencia del Atosiban que tiene alta afinidad por los dos tipos de receptores (Reinheimer, 2007); asî mismo evidencia mayor potencia y duración que el Atosiban (Vrachnis et al., 2011).

\section{El Rol de la Oxitocina en distintos formas de Interacción social}

Estudios en animales no humanos y humanos han demostrado que la OT tiene un rol modulador en una gran variedad de interacciones sociales (Lukas et al., 2011) que han sido relevantes en la evolución de los mamíferos como en el reconocimiento social, el comportamiento sexual, el emparejamiento, el comportamiento parental y la agresión.

\section{Reconocimiento social}

El reconocimiento social se define como la capacidad de reconocer un conespecífico familiar para el establecimiento de todas las relaciones sociales. Éste permite la manifestación de comportamientos adecuados, ya sean de tipo afiliativo (emparejamiento y/o parental) o de tipo agonístico (establecimiento de jerarquías). Estudios con humanos (Domes et al., 2007; Gamer, Zurowski, \& Buchel, 2010) y con roedores (Ferguson, Aldag, Insel, \& Young, 2001) demostraron que la OT en diferentes núcleos de la amígdala es necesaria para el reconocimiento social. En roedores se ha demostrado dimorfismo sexual con respecto al rol de la OT, pues se ha evidenciado que el suministro de agonistas de 
OT facilitan el reconocimiento social en machos y antagonistas interfieren con la habilidad de reconocimiento social en hembras (Bielsky \& Young, 2004).

\section{Comportamiento Sexual}

La OT juega un rol fundamental en la regulación de la conducta sexual tanto en machos como en hembras (Lee et al., 2009). En machos está implicada en: el funcionamiento eréctil (Melis et al., 2010; Succu et al., 2008; Melis et al., 2007), la actividad copulatoria y la eyaculación (Gil, Bhatt, Picotte, \& Hull, 2011). Es importante aclarar que la OT no actúa de forma individual, ésta debe interactuar con otras hormonas como por ejemplo la testosterona, pues si un macho se encuentra castrado a pesar de suministrarle OT no podrá tener erecciones (Connor \& Heithaus, 1996). En hembras la OT facilita: la maduración sexual (Parent et al., 2008), la manifestación de comportamientos de atractividad y receptividad, como por ejemplo en el marcado vaginal que realizan hembras de hámsteres como conducta pre-copulatoria (Martinez, Albers, \& Petrulis, 2010) y/o las respuestas de lordosis (Arletti \& Bertolini, 1985) y la estimulación de la secreción de prolactina en ratas (Kennett \& McKee, 2012).

En humanos se ha identificado que la OT tanto para hombres como para mujeres es un marcador del orgasmo y en el momento de la cópula facilita el transporte de los espermatozoides y el óvulo por el incremento de la contractibilidad de los músculos correspondientes (Burri, Heinrichs, Schedlowski, \& Kruger, 2008). Se ha observado que en mujeres, los niveles de OT en plasma se correlacionan positivamente con la lubricación genital (Salonia et al., 2005). Con lo anterior se observa el rol de la OT a nivel periférico y central en la conducta sexual en mamíferos.

\section{Emparejamiento}

El emparejamiento se caracteriza por la conformación de una pareja en favor de la supervivencia, lo que implica no sólo la reproducción, sino también factores que permitan la prolongación de la especie, como por ejemplo el cuidado parental, la protección contra depredadores y la adaptación a cambios ambientales (Neumann, 2009). Para conocer el rol de la OT en el emparejamiento se han utilizado a roedores monógamos, como los ratones de pradera, y a los campañoles de pino (Young, Gobrogge, Liu, \& Wang, 2011; Wang \& Aragona, 2004) roedores caracterizados por establecer relaciones no monógamas (Wang \& Young, 1997); concluyendo que ratones machos con emparejamiento social monógamo tienden a tener mayor contacto social y preferir a la pareja; mientras que los ratones que no son monógamos permanecen mayor tiempo aislados sin contacto social (Carter \& Keverne, 2009). Estás diferencias están asociadas a la cantidad de receptores de OT en diferentes áreas del cerebro. Así, mientras que los ratones de pradera tienen una densidad alta en la corteza pre-frontal medial (Smeltzer, Curtis, Aragona, \& Wang, 2006), en el núcleo accumbens (Young et al., 2001) y en la amígdala lateral (Insel \& Shapiro, 1992); los campañoles de montaña presentan altas densidades en el septum lateral, el núcleo olfatorio anterior, el septum lateral y algunos núcleo de la amígdala (Parker, Phillips, Kinney, $\&$ Lee, 2001). Es necesario aclarar que existe dimorfismo sexual en la expresión de OT que aún no es claro (de Boer, van Buel \& Ter Host, 2012).

Estudios en la conformación de pareja en humanos sugieren que la OT suministrada intranasal tiene un rol importante en las primeras etapas de la relación romántica. En el estudio de Schneiderman et al. (2012) se compararon personas que se encontraban iniciando relaciones románticas con personas solteras y se observó en imágenes por resonancia magnética funcional (fMRI), un incremento de la actividad del sistema oxitocinérgico mayor en enamorados que en solteros. En otro estudio, Hurlemann et al. (2010) demostraron que la OT facilita la confianza interpersonal y la empatía considerados factores importantes en la formación de pareja. Finalmente, en el estudio de Grewen, Girdler, Amico, y Light (2005) tanto para hombres como para mujeres los niveles de OT en plasma se 
correlacionan con el reporte de apoyo de pareja, entre más alto sientan tener apoyo en su pareja más altos niveles de OT en plasma registraron.

\section{Conducta Parental}

La OT liberada de forma periférica interviene en las contracciones uterinas para el trabajo de parto y en la producción de leche (Neumann, 2008); mientras que la OT liberada en el sistema nervioso central facilita el inicio y mantenimiento de las conductas específicas del cuidado materno (Lee et al., 2009) en diferentes especies. En roedores cuando se inhibe la liberación de OT, las conductas relacionadas al comportamiento materno como la construcción del nido, la lactancia, la limpieza de las crías y la defensa se interrumpen (Leng, Meddle, \& Douglas, 2008). Estudios con ratas sexualmente ingenuas (Pedersen, Ascher, Monroe, \& Prange, Jr., 1982; Pedersen \& Prange, Jr., 1979) demostraron que al suministrarles OT manifiestan conductas maternas específicas similares a las conductas que expresan hembras con crías propias.

Estudios con humanos han demostrado que altos niveles de OT se relacionan con una mejor interacción entre madres-bebés, debido a que la OT permite un reconocimiento rápido por parte de la madre sobre las emociones, estados de ánimo y sensaciones físicas del bebé (Strathearn, Iyengar, Fonagy, \& Kim, 2012). En hombres también se ha observado rol de la OT en la conducta paterna. Naber et al. (2010) demostraron por primera vez en un estudio experimental que al suministrar OT intranasal a padres, éstos tienden a tener interacciones más sensibles y menos hostiles con sus hijos durante el juego.

Es importante mencionar que la OT también se encuentra relacionada a la agresión materna, conducta que se elicita en el momento en que la madre percibe potencial peligro para sus crías. Varios estudios con distintas especies han demostrado una correlación positiva entre la liberación de OT, tanto en el PVN (Bosch et al., 2005) como en el núcleo central de la amígdala
(Ferris et al., 1992), y el comportamiento de agresión evaluado en la prueba de defensa materna, concluyendo que la OT facilita la agresión materna.

\section{Agresión}

El comportamiento agresivo se podría definir como un comportamiento que se manifiesta con la intención de infligir daño o como respuesta de una amenaza de otro individuo (Trainor, Sisk, \& Nelson, 2009). Este comportamiento se presenta en situaciones sociales en las que se establece una jerarquía social dentro de un grupo en función del alimento, el espacio o la pareja o en situaciones de defensa para sobrevivir ante un contrincante (Lee et al., 2009); por lo que se puede clasificar este comportamiento en: agresión antidepredatoria (Blanchard, Blanchard, Rodgers, \& Weiss, 1990), agresión defensiva y ofensiva (Blanchard \& Blanchard, 2010), agresión depredatoria (Knutson \& Hynan, 1973), agresión dominante (Blanchard, Fukunaga-Stinson, Takahashi, Flannelly, \& Blanchard, 1984), agresión maternal (Takahashi \& Lore, 1982), agresión relacionada al comportamiento sexual (Motelica-Heino, Edwards, \& Roffi, 1993) y en agresión territorial (Thurmond, 1975).

Debido a que el comportamiento agresivo es primitivo y altamente conservado entre las diferentes especies de vertebrados, los mecanismos neurales subyacentes son similares inter-especie (Trainor et al., 2009); por lo que los modelos animales, permiten una comprensión adecuada de los mecanismos que subyacen el comportamiento agresivo en los humanos. Por ejemplo, varios biomodelos han permitido identificar que la regulación del comportamiento agresivo está relacionado con áreas límbicas (Trainor et al., 2009), principalmente la activación de la amígdala, la cual se relaciona con distintos tipos de encuentros agresivos en distintas especies: ratas (Vochteloo \& Koolhaas, 1987; Wang, He, Zhao, \& Li, 2013), lagartos (Tarr, 1977), gatos (Zagrodzka \& Fonberg, 1978) y primates (DeFrance \& Hutchinson, 1972). 
Estudios con roedores han mostrado que la agresión es mediada en parte por la OT. DeVries, Young y Nelson (1997) reportaron que ratones con alteración de los genes de OT mostraban menor duración de comportamiento agresivo comparado con ratas tipo salvaje en encuentros agresivos. Engelmann, Ebner, Landgraf, Holsboer y Wotjak (1999) demostraron que al someter a ratas Wistar machos a derrota social, se ocasionaba la liberación de OT dentro del fluido extracelular del NSQ y en la porción ventrolateral anterior del hipotálamo, demostrando que la liberación de OT es activada por el estrés emocional producido por el encuentro agresivo. Neumann (2007) reportó que la exposición a estresores físicos o farmacológicos activan la liberación de OT en el NPV y en el NSO durante pruebas comportamentales como intruso-residente $\mathrm{y}$ nado forzado, y así mismo encontró que hay liberación de OT en la amígdala central y el septum, sugiriendo que la OT es un neuromodulador prometedor para la intervención psicoterapéutica en el tratamiento de enfermedades relacionadas con la ansiedad y la depresión. Bosch et al. (2005) y Ferris et al. (1992) encontraron que si aumentaba el comportamiento agresivo aumentaba la liberación de OT en el NPV y en la amígdala en ratas residentes lactantes cuando se exponían a la prueba de defensa maternal; sin embargo el incremento de la conducta agresiva materna era bloqueada por la administración de antagonistas de OTR; Consiglio, Borsoi, Pereira, y Lucion (2005) reportaron que al suministrar OT en el núcleo medio de la amígdala de ratas que están lactando, inhibía el comportamiento agresivo frente a otras ratas que no están lactando. Finalmente, en el estudio de Litvin et al. (2011) machos Swiss-Webster fueron sometidos a derrota social crónica y a pesar de que no estaban evaluando principalmente los efectos de la OT sino los efectos de la AVP, encontraron que los animales derrotados mostraron mayores niveles de ARNm para OTR en la amígdala y en el septum a diferencia de los controles, sugiriendo que la OT modula comportamientos anti y pro sociales.
Los hallazgos presentados sugieren entonces que el rol de la OT en el comportamiento agresivo podría estar relacionado en mayor medida a las respuestas de estrés frente a estresores sociales que a la agresión por sí misma, lo que indica que posiblemente otros factores asociados a la liberación de OT están relacionados con el encuentro agresivo.

\section{Oxitocina y su Relación con el Estrés Social}

Cuando se presenta un desbalance entre los esfuerzos y las recompensas en las distintas formas de interacción social entre individuos, surge una de las fuentes más importantes de estrés en la vida de los individuos: el estrés social (Tamashiro, Nguyen, \& Sakai, 2005). La principal respuesta al estrés social es a través de la relación del HPA y la liberación simultánea de otras sustancias como la OT. En el estudio de Neumann et al. (2000) para determinar ésta relación se suministró intracerebroventricularmente un antagonista de OT (des Gly-NH2 d(CH2) 5 [Tyr(Me)2, Thr4] OVT) a un grupo de ratas macho y un grupo de ratas hembra expuestas a pruebas de ansiedad como el laberinto en cruz elevado. Los resultados mostraron que al bloquear los receptores de OT, se afectaba la liberación basal de ACTH y corticosterona, aumentando los niveles en plasma sanguíneo, independientemente del género. Para determinar la localización de los efectos de la OT, en el mismo estudio, antagonistas de OTR fueron aplicados en el septum mediolateral, la amígdala y el NPV usando microdiálisis reversa. Los resultados indicaron que solo hubo un aumento significativo en la actividad del eje HPA cuando la acción de la OT fue bloqueada en el NPV (Neumann, 2002). Como conclusión, la inhibición del eje HPA ocurre en parte por la liberación de OT en el NPV.

En otro estudio (Gibbs 1985), se utilizó inmunoneutralización de OT en plasma durante una prueba de suspensión de cola para generar estrés, donde se evidenció una reducción del $59 \%$ en la concentración de ACTH en ratas, 
indicando el rol de la OT en la regulación de la secreción de la ACTH. Finalmente, en el estudio pionero de Windle, Shanks, Lightman y Ingram (1997), se observó que la OT al ser suministrada intracerebroventricularmente disminuye significativamente los niveles de corticosterona cuando ratas Sprague-Dawley eran sometidas a ruidos fuertes $(114 \mathrm{~dB})$. Así mismo encontró que las ratas al ser expuestas a pruebas comportamentales relacionadas a la ansiedad como por ejemplo el laberinto de cruz elevado, la OT tenía efectos de tipo ansiolítico.

Como conclusión de los anteriores hallazgos, se afirma que la OT actúa como inhibidor de la liberación de la hormona de ACTH causando una disminución en las respuestas del eje HPA y por ende una inhibición de los efectos ansiogénicos producidos por la ACTH en diversas situaciones de estrés, por lo que podría pensarse a la OT como un modulador sobre la fisiología del estrés. Es decir, la OT podría ejerce un efecto ansiolítico ante las respuestas de estrés social.

Oxitocina: modulador en la fisiología del estrés social

La OT es conocida por modular funciones neurales relacionadas principalmente a las respuestas fisiológicas de estrés social (Neumann \& Landgraf, 2012). En el estudio realizado por Ring et al. (2006), ratones machos fueron expuestos a tres modelos preclínicos farmacológicamente validados: laberinto elevado en cero, hipertermia inducida por estrés y la prueba de las cuatro placas. Los resultados indicaron que al suministrarles OT las respuestas relacionadas al estrés disminuían en las tres pruebas, sugiriendo a los agonistas de OTR como un nuevo ansiolítico potencialmente útil terapéuticamente. Al igual que en ratones, en ratas también se han confirmado los efectos ansiolíticos de la OT; en el estudio de Slattery y Neumann (2010) se mostró que una cepa de ratas Wistar caracterizada por la presencia de comportamientos relacionados con la ansiedad al recibir una infusión crónica de OT intracerebroventricular, disminuían sus comportamientos ansiosos al ser sometidas a la caja claro/oscuro. Los resultados sugirieron que el suministro de OT es eficaz para atenuar los rasgos altos de ansiedad en modelos de animales. Por otra parte, en el estudio de Amico, Mantella, Vollmer y Li (2004), se evidenció que ratones knock out del gen de OT, al ser sometidos a diferentes pruebas de ansiedad, incluido exposición a ambientes novedosos, mostraron mayores comportamientos asociados a la ansiedad y mayor liberación de corticosterona comparado con ratones de tipo salvaje.

Las propiedades ansiolíticas de la OT están mediadas en parte por su acción en la amígdala y en el NPV (Lee et al., 2009; Neumann \& Landgraf, 2012). En el modelo propuesto por Viviani y Stoop (2008), se demostró que la OT excita neuronas GABAergicas de la amígdala central y lateral, que a su vez inhiben a las neuronas que se encargan de aumentar las respuestas comportamentales del miedo, por lo tanto se concluye que la OT modula las respuestas automáticas de miedo (congelamiento en ratas) por sus efectos en la amígdala (Knobloch et al., 2012; Huber, Veinante, \& Stoop, 2005). De igual forma, algunos estudios han demostrado que la OT en la amígdala central no solo modula respuestas de miedo sino también comportamientos sociales como el comportamiento agresivo (Bosch et al., 2005; Consiglio et al., 2005). En humanos la OT también reduce la activación de la amígdala en respuesta a estímulos amenazantes o de riesgo (al igual que en modelos animales) aumentando la sensación de confianza (Baumgartner, Heinrichs, Vonlanthen, Fischbacher, \& Fehr, 2008).

En el estudio de Lukas et al. (2011), se evalúo la función pro social de la OT como su efecto en la activación de la amígdala, los resultados indicaron que la OT tiene efectos en la preferencia social y en la disminución de los efectos comportamentales (evitación y escape) de un episodio de derrota social, pero no en la activación de la amígdala; concluyendo que la OT podría revertir la evitación social inducida por el estrés y por lo tanto podría ser de uso para el tratamiento de la fobia social y disfunción 
social en los seres humanos. En el estudio de Litvin et al. (2011) identificaron la expresión de ARNm de OTR en la amígdala y el septum lateral de sujetos sometidos a estrés social crónico; sin embargo como el objetivo principal de su estudio fue evaluar la función ansiogénica de la AVP, no evaluaron la relación de la expresión de ARNm de OTR y los posibles efectos ansiolíticos de la OT. A pesar de los resultados de estos recientes estudios, aún se desconoce los efectos pro sociales de la OT en el estrés social crónico, generando un interrogante sobre los posibles efectos ansiolíticos de la OT en este tipo de estrés.

\section{Conclusión}

En el último siglo, la OT ha sido una molécula especialmente estudiada por su relación directa con el comportamiento social, el estrés social y la ansiedad. Las propiedades fisiológicas de la OT han permitido identificar el rol facilitador o inhibidor tanto en el SNP (el cual fue conocido desde inicios del siglo XX) como en el SNC (desde la décadas de los 80's se aumentó el interés investigativo). Dentro de las distintas formas de interacción social en las cuales la OT tiene un efecto modulador como facilitador se encuentran el reconocimiento social, la conducta sexual, el emparejamiento y la conducta parental en distintas especies de animales, incluido el humano. Sin embargo, frente a la agresión (comportamiento agonístico), los efectos moduladores de la OT están dirigidos no al comportamiento agresivo per se, sino a las respuestas de estrés que se presentan en un encuentro agonístico; lo que conlleva a aumentar el interés investigativo de los efectos del estrés social en la liberación o inhibición de esta hormona nanopéptida.

Distintos estudios presentados en esta revisión, reconocieron a la OT como un potencial inhibidor de las respuestas del eje HPA, a través de la inhibición de la liberación de la hormona ACTH, en algunos escenarios de estrés: agudo y episódico. Concluyendo que la OT podría utilizarse como un ansiolítico prometedor. Sin embargo, aún no es claro cuáles son los efectos del estrés social crónico en la liberación de la OT. Por lo tanto, se recomienda llevar a cabo estudios que permitan identificar cómo la OT podría funcionar como un modulador efectivo en el tratamiento de distintos tipos de trastornos asociados a la ansiedad (inducida por el estrés social crónico), como por ejemplo la fobia social y la disfunción social en humanos; pues como lo menciona la Sociedad Americana de Psicología (APA), solo en Estados Unidos, al menos 19 millones de adultos padecen este tipo de trastorno.

\section{Referencias}

Amico, J. A., Mantella, R. C., Vollmer, R. R., \& Li, X. (2004). Anxiety and stress responses in female oxytocin deficient mice. J.Neuroendocrinol., 16 (4), 319-324.10.1111/ j.0953-8194.2004.01161.x [doi];JNE1161 [pii]

Arletti, R. \& Bertolini, A. (1985). Oxytocin stimulates lordosis behavior in female rats. Neuropeptides, 6 (3), 247-253.

Bales, K. L. \& Perkeybile, A. M. (2012). Developmental experiences and the oxytocin receptor system. Horm.Behav., 61 (3), 313-319. S0018-506X(11)00290-X [pii];10.1016/j.yhbeh.2011.12.013 [doi]

Baumgartner, T., Heinrichs, M., Vonlanthen, A., Fischbacher, U., \& Fehr, E. (2008). Oxytocin Shapes the Neural Circuitry of Trust and Trust Adaptation in Humans. Neuron, 58 (4), 639-650.

Bethlehem, R. A. I., van Honk, J., Auyeung, B., \& Baron-Cohen, S. (2013). Oxytocin, brain physiology, and functional connectivity: A review of intranasal oxytocin fMRI studies. Psychoneuroendocrinology, 38 (7), 962-974.

Bielsky, I. F. \& Young, L. J. (2004). Oxytocin, vasopressin, and social recognition in mammals. Peptides, 25 (9), 1565-1574.

Blanchard, D. C. \& Blanchard, R. J. (2010). Offensive and Defensive Aggression. In F.K.Editors-in-Chief:-á-áGeorge, Michel Le Moal and Richard, \& F. T. Richard (Eds.), 
Encyclopedia of Behavioral Neuroscience (pp. 484-489). Oxford: Academic Press.

Blanchard, D. C., Fukunaga-Stinson, C., Takahashi, L. K., Flannelly, K. J., \& Blanchard, R. J. (1984). Dominance and aggression in social groups of male and female rats. Behavioural Processes, 9 (1), 31-48.

Blanchard, R. J., Blanchard, D. C., Rodgers, J., \& Weiss, S. M. (1990). The characterization and modelling of antipredator defensive behavior. Neuroscience $\mathfrak{E}$ Biobehavioral Reviews, 14 (4), 463-472.

Bosch, O. J., Meddle, S. L., Beiderbeck, D. I., Douglas, A. J., \& Neumann, I. D. (2005). Brain oxytocin correlates with maternal aggression: link to anxiety. J.Neurosci., 25 (29), 6807-6815.25/29/6807 [pii];10.1523/ JNEUROSCI.1342-05.2005 [doi]

Burri, A., Heinrichs, M., Schedlowski, M., \& Kruger, T. H. C. (2008). The acute effects of intranasal oxytocin administration on endocrine and sexual function in males. Psychoneuroendocrinology, 33 (5), 591-600.

Caldwell, J. D., Walker, C. H., Pedersen, C. A., \& Mason, G. A. (1993). Sexual activity decreases oxytocin receptor densities in the thymus. Life Sci., 52 (22), 1781-1786.

Carter, C. S., DeVries, A. C., \& Getz, L. L. (1995). Physiological substrates of mammalian monogamy: the prairie vole model. Neurosci.Biobehav.Rev., 19 (2), 303-314.014976349400070H [pii]

Carter, C. S. \& Keverne, E. B. (2009). 4 - The Neurobiology of Social Affiliation and Pair Bonding. In W.P.Editors-in-Chief: Donald, P. A. Arthur, Susan E.Fahrbach, M. E. a. R. Anne, \& T. R. Robert (Eds.), Hormones, Brain and Behavior (Second Edition) (pp. 137-166). San Diego: Academic Press.

Connor, R. C. \& Heithaus, M. R. (1996). Approach by great white shark elicits flight response in bottlenose dolphins. Marine Mammal Science, 12 602-606.

Consiglio, A. R., Borsoi, A., Pereira, G. A. M., \& Lucion, A. B. (2005). Effects of oxytocin microinjected into the central amygdaloid nucleus and bed nucleus of stria terminalis on maternal aggressive behavior in rats. Physiology $\mathcal{E}$ Behavior, 85 (3), 354-362.

de Boer, A., van Buel, E. M., \& Ter Horst, G. J. (2012). Love is more than just a kiss: a neurobiological perspective on love and affection. Neuroscience, 201 (0), 114-124.

DeFrance, J. F. \& Hutchinson, R. R. (1972). Electrographic changes in the amygdala and hippocampus associated with biting attack. Physiology $\mathbb{E}$ Behavior, 9 (1), 83-88.

DeVries, A. C., Young, W. S., \& Nelson, R. J. (1997). Reduced aggressive behaviour in mice with targeted disruption of the oxytocin gene. J.Neuroendocrinol., 9 (5), 363-368.

Domes, G., Heinrichs, M., Gl+ñscher, J., B+ +chel, C., Braus, D. F., \& Herpertz, S. C. (2007). Oxytocin Attenuates Amygdala Responses to Emotional Faces Regardless of Valence. Biological Psychiatry, 62 (10), 1187-1190.

Engelmann, M., Ebner, K., Landgraf, R., Holsboer, F., \& Wotjak1 CT (1999). Emotional stress triggers intrahypothalamic but not peripheral release of oxytocin in male rats. J.Neuroendocrinol., 11 (11), 867-872.jne403 [pii]

Ferguson, J. N., Aldag, J. M., Insel, T. R., \& Young, L. J. (2001). Oxytocin in the medial amygdala is essential for social recognition in the mouse. J.Neurosci., 21 (20), 8278-8285.21/20/8278 [pii]

Ferris, C. F., Foote, K. B., Meltser, H. M., Plenby, M. G., Smith, K. L., \& Insel, T. R. (1992). Oxytocin in the amygdala facilitates maternal aggression. Ann.N.Y.Acad.Sci., 652 456-457.

Gamer, M., Zurowski, B., \& Buchel, C. (2010). Different amygdala subregions mediate valence-related and attentional effects of oxytocin in humans. Proc.Natl.Acad.Sci.U.S.A, 107 (20), 9400-9405.1000985107 [pii];10.1073/ pnas. 1000985107 [doi]

Gibbs, D. M. (1985). Immunoneutralization of oxytocin attenuates stress-induced corticotropin secretion in the rat. Regulatory Peptides, 12 (4), 273-277. 
Gil, M., Bhatt, R., Picotte, K. B., \& Hull, E. M. (2011). Oxytocin in the medial preoptic area facilitates male sexual behavior in the rat. Hormones and Behavior, 59 (4), 435-443.

Gimpl, G. \& Fahrenholz, F. (2001). The oxytocin receptor system: structure, function, and regulation. Physiol Rev., 81 (2), 629-683.

Grewen, K. M., Girdler, S. S., Amico, J., \& Light, K. C. (2005). Effects of partner support on resting oxytocin, cortisol, norepinephrine, and blood pressure before and after warm partner contact. Psychosom.Med., 67 (4), 531-538.67/4/531 [pii];10.1097/01.psy.0000170341.88395.47 [doi]

Huber, D., Veinante, P., \& Stoop, R. (2005). Vasopressin and oxytocin excite distinct neuronal populations in the central amygdala. Science, $308 \quad$ (5719), 245-248.308/5719/245 [pii];10.1126/science.1105636 [doi]

Hurlemann, R., Patin, A., Onur, O. A., Cohen, M. X., Baumgartner, T., Metzler, S. et al. (2010). Oxytocin enhances amygdala-dependent, socially reinforced learning and emotional empathy in humans. J.Neurosci., 30 (14), 4999-5007.30/14/4999 [pii];10.1523/ JNEUROSCI.5538-09.2010 [doi]

Insel, T. R. \& Shapiro, L. E. (1992). Oxytocin receptor distribution reflects social organization in monogamous and polygamous voles. Proc.Natl.Acad.Sci.U.S.A, $89 \quad$ (13), 5981-5985.

Kennett, J. E. \& McKee, D. T. (2012). Oxytocin: an emerging regulator of prolactin secretion in the female rat. J.Neuroendocrinol., 24 (3), 403-412.10.1111/ j.1365-2826.2011.02263.x [doi]

Kiss, A. \& Mikkelsen, J. D. (2005). Oxytocin-anatomy and functional assignments: a minireview. Endocr.Regul., 39 (3), 97-105.

Knobloch, H., Charlet, A., Hoffmann, L., Eliava, M., Khrulev, S., Cetin, A. et al. (2012). Evoked Axonal Oxytocin Release in the Central Amygdala Attenuates Fear Response. Neuron, 73 (3), 553-566.

Knutson, J. F. \& Hynan, M. T. (1973). Predatory aggression and irritable aggression: Shockinduced fighting in mouse-killing rats. Physiology Eु Behavior, 11 (1), 113-115.

Lee, H. J., Macbeth, A. H., Pagani, J. H., \& Scott, Y. I. (2009). Oxytocin: The great facilitator of life. Progress in Neurobiology, 88 (2), 127-151.

Leng, G., Meddle, S. L., \& Douglas, A. J. (2008). Oxytocin and the maternal brain. Current Opinion in Pharmacology, 8 (6), 731-734.

Litvin, Y., Murakami, G., \& Pfaff, D. W. (2011). Effects of chronic social defeat on behavioral and neural correlates of sociality: Vasopressin, oxytocin and the vasopressinergic V1b receptor. Physiology $\mathbb{E}$ Behavior, 103 (3GÇô4), 393-403.

Loup, F., Tribollet, E., Dubois-Dauphin, M., \& Dreifuss, J. J. (1991). Localization of high-affinity binding sites for oxytocin and vasopressin in the human brain. An autoradiographic study. Brain Res., 555 (2), 220-232.0006-8993(91)90345-V [pii]

Loup, F., Tribollet, E., Dubois-Dauphin, M., Pizzolato, G., \& Dreifuss, J. J. (1989). Localization of oxytocin binding sites in the human brainstem and upper spinal cord: an autoradiographic study. Brain Res., 500 (1-2), 223-230.0006-8993(89)90317-X [pii]

Lowbridge, J., Manning, M., Haldar, J., \& Sawyer, W. H. (1977). Synthesis and some pharmacological properties of [4-threonine, 7-glycine]oxytocin, [1-(L-2-hydroxy-3mercaptopropanoic acid), 4-threonine, 7glycine]oxytocin (hydroxy[Thr4, Gly7]oxytocin), and [7-Glycine]oxytocin, peptides with high oxytocic-antidiuretic selectivity. J.Med.Chem., 20 (1), 120-123.

Ludwig, M. \& Leng, G. (2006). Dendritic peptide release and peptide-dependent behaviours. Nat.Rev.Neurosci., 7 (2), 126-136.nrn1845 [pii];10.1038/nrn1845 [doi]

Lukas, M., Toth, I., Reber, S. O., Slattery, D. A., Veenema, A. H., \& Neumann, I. D. (2011). The neuropeptide 
oxytocin facilitates pro-social behavior and prevents social avoidance in rats and mice. Neuropsychopharmacology, 36 (11), 2159-2168.npp201195 [pii];10.1038/ npp.2011.95 [doi]

Manning, M., Lowbridge, J., Sawyer, W. H., \& Haldar, J. (1976). Synthesis and some pharmacological properties of [1-(L-2-hydroxy-3-mercaptopropanoic acid), 4-threonine]oxytocin (hydroxy [4-thr] oxytocin), a peptide with strikingly high oxytocic potency and of [1-(L-2-hydroxy-3-mercaptopropanoic acid) ]oxytocin (hydroxy-oxytocin). J.Med.Chem., 19 (3), 376-380.

Manning, M., Misicka, A., Olma, A., Bankowski, K., Stoev, S., Chini, B. et al. (2012). Oxytocin and vasopressin agonists and antagonists as research tools and potential therapeutics. J.Neuroendocrinol., 24 (4), 609-628.10.1111/ j.1365-2826.2012.02303.x [doi]

Manning, M., Stoev, S., Chini, B., Durroux, T., Mouillac, B., \& Guillon, G. (2008). Peptide and non-peptide agonists and antagonists for the vasopressin and oxytocin V1a, V1b, V2 and OT receptors: research tools and potential therapeutic agents. Prog.Brain Res., 170 473-512.S0079-6123(08)00437-8 [pii];10.1016/S0079-6123(08)00437-8 [doi]

Martinez, L. A., Albers, H. E., \& Petrulis, A. (2010). Blocking oxytocin receptors inhibits vaginal marking to male odors in female Syrian hamsters. Physiol Behav., 101 (5), 685-692.S0031-9384(10)00292-1 [pii];10.1016/j.physbeh.2010.08.007 [doi]

Melis, M. R., Melis, T., Cocco, C., Succu, S., Sanna, F., Pillolla, G. et al. (2007). Oxytocin injected into the ventral tegmental area induces penile erection and increases extracellular dopamine in the nucleus accumbens and paraventricular nucleus of the hypothalamus of male rats. Eur.J.Neurosci., 26 (4), 1026-1035.EJN5721 [pii];10.1111/ j.1460-9568.2007.05721.x [doi]
Melis, M. R., Succu, S., Cocco, C., Caboni, E., Sanna, F., Boi, A. et al. (2010). Oxytocin induces penile erection when injected into the ventral subiculum: role of nitric oxide and glutamic acid. Neuropharmacology, 58 (7), 1153-1160.S0028-3908(10)00041-9 [pii];10.1016/j.neuropharm.2010.02.008 [doi]

Motelica-Heino, I., Edwards, D. A., \& Roffi, J. (1993). Intermale aggression in mice: Does hour of castration after birth influence adult behavior? Physiology $\mathcal{E}$ Behavior, 53 (5), 1017-1019.

Naber, F., van IJzendoorn, M. H., Deschamps, P., van Engeland, H., \& BakermansKranenburg, M. J. (2010). Intranasal oxytocin increases fathersGÇÖ observed responsiveness during play with their children: A double-blind within-subject experiment. Psychoneuroendocrinology, 35 (10), 1583-1586.

Neumann, I. D. (2007). Stimuli and consequences of dendritic release of oxytocin within the brain. Biochem.Soc.Trans., 35 (Pt 5), 1252-1257.BST0351252 [pii];10.1042/ BST0351252 [doi]

Neumann, I. D. (2008). Brain oxytocin: a key regulator of emotional and social behaviours in both females and males. J.Neuroendocrinol., 20 (6), 858-865.JNE1726 [pii];10.1111/ j.1365-2826.2008.01726.x [doi]

Neumann, I. D. \& Landgraf, R. (2012). Balance of brain oxytocin and vasopressin: implications for anxiety, depression, and social behaviors. Trends Neurosci., 35 (11), 649-659.S0166-2236(12)00152-X [pii];10.1016/j.tins.2012.08.004 [doi]

Neumann, I. D., Wigger, A., Torner, L., Holsboer, F., \& Landgraf, R. (2000). Brain oxytocin inhibits basal and stress-induced activity of the hypothalamo-pituitaryadrenal axis in male and female rats: partial action within the paraventricular nucleus. J.Neuroendocrinol., 12 (3), 235-243.jne442 [pii] 
Neumann, I. D. (2002). Chapter 12 Involvement of the brain oxytocin system in stress coping: interactions with the hypothalamopituitary-adrenal axis. In D.Poulain (Ed.), Progress in Brain Research Vasopressin and Oxytocin: From Genes to Clinical Applications (Volume 139 ed., pp. 147-162). Elsevier.

Neumann, I. D. (2009). The advantage of social living: Brain neuropeptides mediate the beneficial consequences of sex and motherhood. Frontiers in Neuroendocrinology, 30 (4), 483-496.

Ostrowski, N. L. (1998). Oxytocin receptor mRNA expression in rat brain: implications for behavioral integration and reproductive success. Psychoneuroendocrinology, 23 (8), 989-1004.S0306-4530(98)00070-5 [pii]

Parent, A. S., Rasier, G., Matagne, V., Lomniczi, A., Lebrethon, M. C., Gerard, A. et al. (2008). Oxytocin facilitates female sexual maturation through a glia-to-neuron signaling pathway. Endocrinology, 149 (3), 1358-1365.en.2007-1054][pii];10.1210/ en.2007-1054 [doi]

Parker, K. J., Phillips, K. M., Kinney, L. F., \& Lee, T. M. (2001). Day length and sociosexual cohabitation alter central oxytocin receptor binding in female meadow voles (Microtus pennsylvanicus). Behav.Neurosci., 115 (6), 1349-1356.

Pedersen, C. A., Ascher, J. A., Monroe, Y. L., \& Prange, A. J., Jr. (1982). Oxytocin induces maternal behavior in virgin female rats. Science, 216 (4546), 648-650.

Pedersen, C. A. \& Boccia, M. L. (2002). Oxytocin maintains as well as initiates female sexual behavior: effects of a highly selective oxytocin antagonist. Horm.Behav., 41 (2), 170-177.10.1006/hbeh.2001.1736 [doi];S0018506X01917362 [pii]

Pedersen, C. A., Caldwell, J. D., Peterson, G., Walker, C. H., \& Mason, G. A. (1992). Oxytocin activation of maternal behavior in the rat. Ann.N.Y.Acad.Sci., 652 58-69.

Pedersen, C. A. \& Prange, A. J., Jr. (1979). Induction of maternal behavior in virgin rats after intracerebroventricular administration of oxytocin. Proc.Natl.Acad.Sci.U.S.A, 76 (12), 6661-6665.

Pedersen, C. A. \& Prange, A. J., Jr. (1985). Oxytocin and mothering behavior in the rat. Pharmacol.Ther., 28 (3), 287-302.0163-7258(85)90056-7 [pii]

Pierzynski, P. (2011). Oxytocin and vasopressin $\mathrm{V}(1 \mathrm{~A})$ receptors as new therapeutic targets in assisted reproduction. Reprod.Biomed.Online., 22 (1), 9-16.S1472-6483(10)00640-1 [pii];10.1016/j.rbmo.2010.09.015 [doi]

Pierzynski, P., Reinheimer, T. M., \& Kuczynski, W. (2007). Oxytocin antagonists may improve infertility treatment. Fertil.Steril., 88 (1), 213-222.S0015-0282(07)00273-7 [pii];10.1016/j.fertnstert.2006.09.017 [doi]

Reinheimer, T. M. (2007). Barusiban suppresses oxytocin-induced preterm labour in non-human primates. BMC.Pregnancy.Childbirth., 7 Suppl 1 S15.1471-2393-7-S1-S15

[pii];10.1186/1471-2393-7-S1-S15 [doi]

Ring, R. H., Malberg, J. E., Potestio, L., Ping, J., Boikess, S., Luo, B. et al. (2006). Anxiolytic-like activity of oxytocin in male mice: behavioral and autonomic evidence, therapeutic implications. Psychopharmacology (Berl), 185 (2), 218-225.10.1007/s00213-005-0293-z [doi]

Ring, R. H., Schechter, L. E., Leonard, S. K., Dwyer, J. M., Platt, B. J., Graf, R. et al. (2010). Receptor and behavioral pharmacology of WAY-267464, a non-peptide oxytocin receptor agonist. Neuropharmacology, 58 (1), 69-77.S0028-3908(09)00224X [pii];10.1016/j.neuropharm.2009.07.016 [doi]

Ross, H. E. \& Young, L. J. (2009). Oxytocin and the neural mechanisms regulating social cognition and affiliative behavior. Frontiers in Neuroendocrinology, 30 (4), 534-547.

Sabatier, N. (2006). alpha-Melanocytestimulating hormone and oxytocin: a peptide signalling cascade in the hypothalamus. J.Neuroendocrinol., 
18 (9), 703-710.JNE1464 [pii];10.1111/ j.1365-2826.2006.01464.x [doi]

Salonia, A., Nappi, R. E., Pontillo, M., Daverio, R., Smeraldi, A., Briganti, A. et al. (2005). Menstrual cycle-related changes in plasma oxytocin are relevant to normal sexual function in healthy women. Hormones and Behavior, 47 (2), 164-169.

Schneiderman, I., Zagoory-Sharon, O., Leckman, J. F., \& Feldman, R. (2012). Oxytocin during the initial stages of romantic attachment: Relations to couplesGÇÖ interactive reciprocity. Psychoneuroendocrinology, 37 (8), 1277-1285.

Slattery, D. A. \& Neumann, I. D. (2010). Chronic icv oxytocin attenuates the pathological high anxiety state of selectively bred Wistar rats. Neuropharmacology, 58 (1), 56-61.

Smeltzer, M. D., Curtis, J. T., Aragona, B. J., \& Wang, Z. (2006). Dopamine, oxytocin, and vasopressin receptor binding in the medial prefrontal cortex of monogamous and promiscuous voles. Neuroscience Letters, 394 (2), 146-151.

Strathearn, L., Iyengar, U., Fonagy, P., \& Kim, S. (2012). Maternal oxytocin response during motherGÇôinfant interaction: Associations with adult temperament. Hormones and Behavior, 61 (3), 429-435.

Succu, S., Sanna, F., Cocco, C., Melis, T., Boi, A., Ferri, G. L. et al. (2008). Oxytocin induces penile erection when injected into the ventral tegmental area of male rats: role of nitric oxide and cyclic GMP. Eur.J.Neurosci., 28 (4), 813-821.EJN6385 [pii];10.1111/ j.1460-9568.2008.06385.x [doi]

Takahashi, L. K. \& Lore, R. K. (1982). Intermale and maternal aggression in adult rats tested at different ages. Physiology $\mathcal{E}$ Behavior, 29 (6), 1013-1018.

Tamashiro, K. L. K., Nguyen, M. M. N., \& Sakai, R. R. (2005). Social stress: From rodents to primates. Frontiers in Neuroendocrinology, 26 (1), 27-40.

Tarr, R. S. (1977). Role of the amygdala in the intraspecies aggressive behavior of the iguanid lizard, Sceloporus occidentalis. Physiology EO Behavior, 18 (6), 1153-1158.

Thurmond, J. B. (1975). Technique for producing and measuring territorial aggression using laboratory mice. Physiology $\mathcal{E}$ Behavior, 14 (6), 879-881.

Trainor, B. C., Sisk, C. L., \& Nelson, R. J. (2009). 5 - Hormones and the Development and Expression of Aggressive Behavior. In W.P.Editors-in-Chief: Donald, P. A. Arthur, Susan E.Fahrbach, M. E. a. R. Anne, \& T. R. Robert (Eds.), Hormones, Brain and Behavior (Second Edition) (pp. 167-205). San Diego: Academic Press.

Veinante, P. \& Freund-Mercier, M. J. (1997). Distribution of oxytocin- and vasopressinbinding sites in the rat extended amygdala: a histoautoradiographic study. J.Comp Neurol., 383 (3), 305-325.10.1002/ (SICI) 1096-9861(19970707)383:3<305::AID $\mathrm{CNE} 3>3.0 . \mathrm{CO} ; 2-7$ [pii]

Viero, C., Shibuya, I., Kitamura, N., Verkhratsky, A., Fujihara, H., Katoh, A. et al. (2010). REVIEW: Oxytocin: Crossing the bridge between basic science and pharmacotherapy. CNS.Neurosci.Ther., 16 (5), e138-e156.CNS185 [pii];10.1111/ j.1755-5949.2010.00185.x [doi]

Viviani, D. \& Stoop, R. (2008). Opposite effects of oxytocin and vasopressin on the emotional expression of the fear response. In D.N.a.R.Inga (Ed.), Progress in Brain Research

Vochteloo, J. D. \& Koolhaas, J. M. (1987). Medial amygdala lesions in male rats reduce aggressive behavior: interference with experience. Physiology $\mathcal{E}$ Behavior, 41 (2), 99-102.

Vrachnis, N., Malamas, F. M., Sifakis, S., Deligeoroglou, E., \& Iliodromiti, Z. (2011). The oxytocin-oxytocin receptor system and its antagonists as tocolytic agents. Int.J.Endocrinol., 2011 350546.10.1155/2011/350546 [doi]

Wang, Y., He, Z., Zhao, C., \& Li, L. (2013). Medial amygdala lesions modify aggressive behavior and immediate early gene expression in oxytocin and 
vasopressin neurons during intermale exposure. Behavioural Brain Research, 245 (0), 42-49.

Wang, Z. \& Aragona, B. J. (2004). Neurochemical regulation of pair bonding in male prairie voles. Physiology $\mathcal{E}$ Behavior, 83 (2), 319-328.

Wang, Z. \& Young, L. J. (1997). Ontogeny of oxytocin and vasopressin receptor binding in the lateral septum in prairie and montane voles. Developmental Brain Research, 104 (1GÇô2), 191-195.

Windle, R. J., Shanks, N., Lightman, S. L., \& Ingram, C. D. (1997). Central oxytocin administration reduces stressinduced corticosterone release and anxiety behavior in rats. Endocrinology, 138 (7), 2829-2834.

Yoshimura, R., Kimura, T., Watanabe, D., \& Kiyama, H. (1996). Differential expression of oxytocin receptor mRNA in the developing rat brain. Neurosci.Res., 24 (3), 291-304.0168-0102(95)01003-3 [pii]

Young, K. A., Gobrogge, K. L., Liu, Y., \& Wang, Z. (2011). The neurobiology of pair bonding: Insights from a socially monogamous rodent. Frontiers in Neuroendocrinology, 32 (1), 53-69.

Young, L. J., Lim, M. M., Gingrich, B., \& Insel, T. R. (2001). Cellular Mechanisms of Social Attachment. Hormones and Behavior, 40 (2), 133-138.

Zagrodzka, J. \& Fonberg, E. (1978). Predatory versus alimentary behavior after amygdala lesions in cats. Physiology $\mathbb{E}$ Behavior, 20 (5), 523-531.

\section{Notas}

* Artículo de revisión. Resultado de investigación. 